\title{
Uncovering the sexual tricks of orchid flowers
}

\author{
JULIANNE D. LIVINGSTON
}

\section{Abstract}

Rather than the conventional offer of a food reward such as nectar or pollen, many orchid species from the Australian Chiloglottis genera use sexual deception to attract male pollinators. These orchids emit an odorous pheromone very similar to the sexual pheromone produced by females of the pollinator species, thereby luring the male to the flower with the false offer of sex. The high degree of specificity between sexually deceptive orchids and their pollinators indicates that there must be subtle but important differences in the pheromones produced among even closely related orchids. This paper investigates whether the PACPT (Palmitoyl-Acyl Carrier Protein Thioesterase) gene, found to be highly expressed in the pheromone-producing part of Chilogottis orchids, may be involved in the production of sexual mimicry pheromones. An analysis of the distribution of genetic variation in the gene sequence, as well as its possible effects on the amino acid it encodes was conducted across five species within the Chiloglottis genera. The results are not only highly encouraging for future research into the role of this gene in pheromone production, but may also provide helpful diagnostic tools for cryptic species that pervade the sexually-deceptive orchids. 


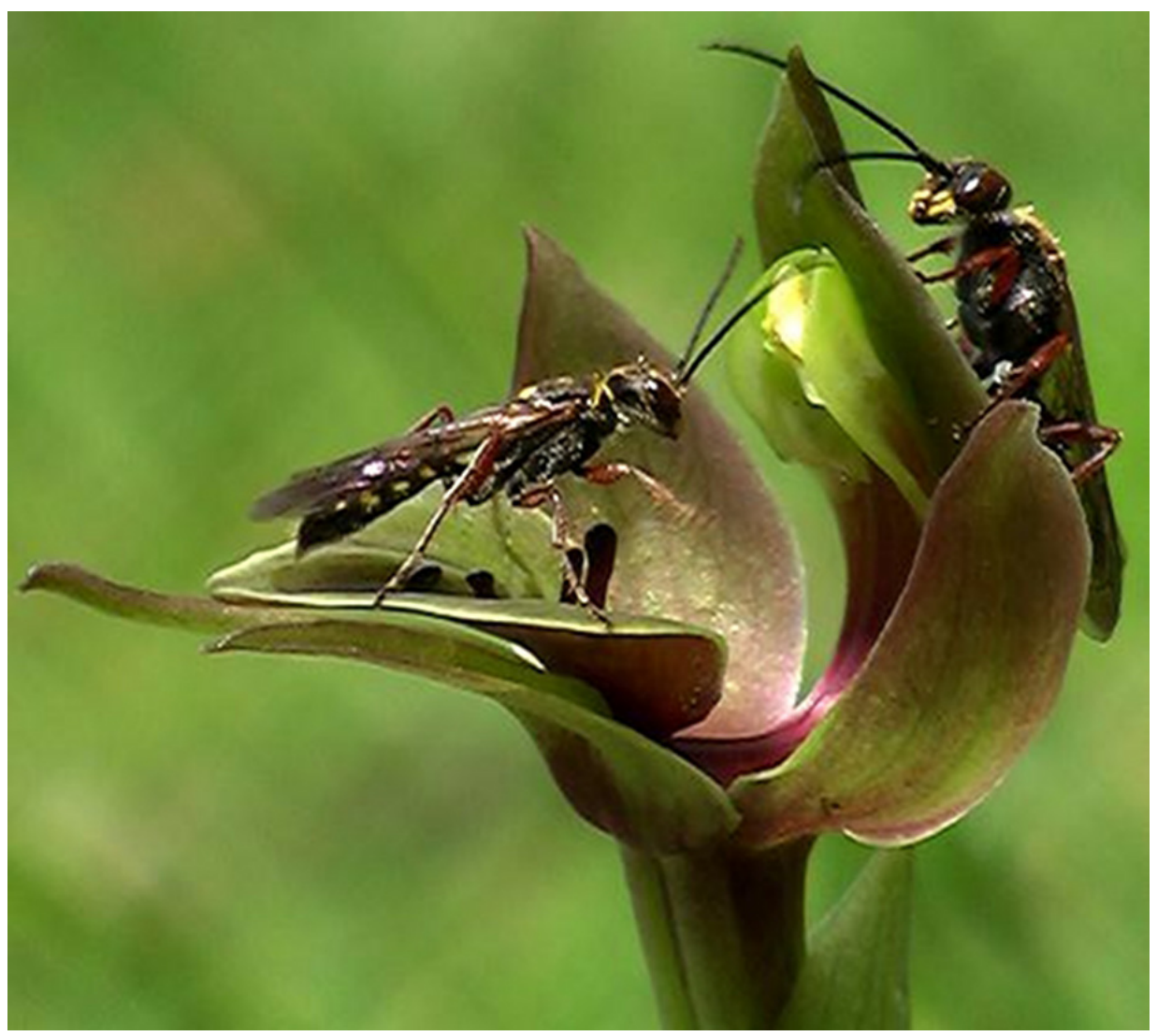

Figure 1. Chiloglottis valida with sexually attracted pollinators.

Credit: Rod Peakall.

Sexual deception of pollinators is surprisingly common and diverse in terrestrial orchids, being the method of pollination in nine of thirty-nine Australian orchid genera within the Diuridea tribe (Dafni \& Bernhardt 1990; Mant et al., 2002; Schiestl \& Peakall 2005). By mimicking the morphology and sex pheromones of female pollinators, these sexually deceptive orchids are able to lure male pollinators with the false offer of sex and accomplish cross-pollination without the cost of a food reward (Schiestl et al., 2003; Bower \& Brown 2009). This type of sexually deceptive pollination has been well studied in many Orchidaceae families (Dafni \& Bernhardt 1990; Soliva \& Widmer 2003; Vela et al., 2007; Mant, Peakall \& Schiestl 2005), with extreme specificity between orchid and pollinator species the norm. Recent investigations into the sexual deception of Neozeleboria (Tiphiidae) thynnine wasp by orchids in the Australian Chiloglottis genera have also revealed very species-specific pollination patterns, such that 
closely related Chiloglottis species are usually pollinated by closely related thynnine wasp species (figure 1) (Mant et al., 2002; Schiestl \& Peakall 2005; Bower \& Brown 2009).

Attraction of specific pollinator species maintains the species borders of sexually deceptive orchids and is accomplished through variation in pheromone traits between species (Mant, Peakall \& Schiestl 2005). The array of odour compounds involved in the mimicry of thynnine wasp sex pheromones have only recently begun to be discovered within the Chiloglottis genera (Schiestl \& Peakall 2005). For instance, a single chemical compound termed 'Chiloglottone' has recently been found to be the sole sex pheromone responsible for the attraction of male pollinators in two sister thynnine wasp species (Schiestl et al., 2003). Interestingly, this pheromone is produced by two distantly related orchid species; Chiloglottis trapeziformis and Chiloglottis valida (Schiestl et al., 2003). The joint production and attraction to the Chiloglottone pheromone in the wasps can easily be explained by a shared ancestry, however, the mimicry in distantly related orchids has raised interestingly evolutionary questions regarding the extent to which compounds involved in pheromone production vary among Chiloglottis orchid species (Mant, Peakall \& Schiestl 2005). This variation and flexibility in pollinator pheromone mimicry may explain the lack of genetic differentiation among Chiloglottis species within subgeneric clades (compared to among clades) i.e. the joint attraction of the same thynnine wasp pollinator by $C$. trapeziformis and $C$. valida has led to gene flow and low genetic variation between the orchid species (Mant, Peakall \& Weston, 2005).

To fully understand the evolutionary processes involved in the mimicry of pollinator sex pheromones by Chiloglottis orchids and the subsequent effects on gene flow and pollinator specificity, the active compounds involved in pheromone production across species and populations must be identified (Mant, Peakall \& Schiestl, 2005). A cDNA library has been constructed containing nucleotide gene sequences which are highly expressed in the pheromoneproducing labellum of Chiloglottis orchids (Schiestl \& Peakall, 2005). Analysis of the taxonomic variation in these gene sequences will assist in identifying the multiple genes involved in pheromone production and the subsequent variation that results in subtle but important differences in the pheromones produced by different Chiloglottis orchids. This paper aims to measure the genetic variation in the exon and intron nucleotide sequences of the highly expressed PalmitoylAcyl Carrier Protein Thioesterase (PACPT) gene across five distantly related Chiloglottis species. The protein encoded in this gene is known to play a crucial role in the biosynthesis of plant fatty acids (Jones et al., 1995), hence any genetic variation within the expressed exon sequence that results in nonsynonymous changes between species in the encoded amino acid sequence may alter the biosynthesis pathway of the sex pheromones. 
A secondary aim of this investigation into genetic variation is the development of primers for highly variable intron sequences within the Chiloglottis genera. Species-specific variation is expected to occur most highly within the intron sequences of the PACPT gene as these are relatively isolated from selection. As a result, introns accumulate single nucleotide polymorphisms (SNPs) and nucleotide insertions and deletions (indels) more frequently than exons (Campbell et al., 2006). Therefore, identification of species-specific variation in intron sequences may not only complement existing markers for phylogenetic analysis of Chiloglottis orchids, but also aid in the identification of morphologically similar or cryptic orchid species (such as Chiloglottis valida subsp.) that have so far confounded and limited many studies on sexually deceptive orchid-pollinator interactions (Mant, Peakall, \& Schiestl, 2005; Mant, Peakall, \& Weston, 2005; Bower \& Brown 2009).

\section{Methods}

\section{Primer Design}

Primers were designed using web-based PRIMER 3 software (Rozen \& Skaletsky 2000) to amplify the gene sequence encoding for the production of palmitoylacyl carrier protein thioesterase $(P A C P T)$ in five Chiloglottis species based on a consensus cDNA sequence of PACPT from $C$. trapeziformis (Purdue Genomics Facility, 2010). I predicted the location of four introns in the cDNA sequence (exons only) by comparison with the complete genomic sequence of PACPT in the genomic model plant Arabidopsis (GenBank). The four sets of primers were designed to amplify and sequence four overlapping sections of the PACPT gene sequence, avoiding intron/exon junctions. The total exon length was estimated to be around 1362 nucleotide bases with four introns estimated to be between 30 and 150 bases in length each.

\section{Orchid DNA sequencing}

Each of the five Chiloglottis species included in this study, has a distinct pollinator that is distinguishable by morphological characteristics (Mant et al., 2002). A simplified phylogenetic tree of the Chiloglottis genera (Mant, Peakall, \& Weston, 2005), illustrating the placement of the five sampled Chiloglottis species across the three main clades is presented in figure 2. Multiple species samples were included to allow comparison of genetic variation among and within species and clades (table 1). 
Orchid DNA was extracted using DNeasy Plant Mini Kit (Qiagen, Melbourne, Australia), with confirmation of DNA extract obtained by gel electrophoresis ( $1 \%$ agarose, $40 \mathrm{~min}$ at 110 volts). Polymerase chain reactions (PCRs) (40 $\mu \mathrm{l})$ contained: $24.9 \mu \mathrm{l}$ milliQ $\mathrm{H}_{2} 0 ; 4.0 \mu \mathrm{l}$ 10X PCR buffer (Qiagen, containing 15 $\mathrm{mM} \mathrm{MgCl}{ }_{2}$ ): $2.5 \mathrm{mM}$ dNTPs, $2.0 \mu \mathrm{l} ; 25 \mathrm{mM} \mathrm{MgCl}_{2}, 2.4 \mu \mathrm{l}$ (Qiagen); $1 \%$ (w/v) BSA(Biolabs), $0.5 \mu \mathrm{l} 2 \mathrm{mM}$ of each primer in a pair, $2.0 \mu \mathrm{l} ; 5$ units/ $\mu \mathrm{l} \mathrm{Taq}$ polymerase, $0.2 \mu \mathrm{l}$; and $100 \mathrm{~nm} / \mu \mathrm{l}$ of template DNA; $2.0 \mu \mathrm{l}$.

\begin{tabular}{|l|l|}
\hline Taxon & Location \\
\hline C. diphylla & NSW: Buladelah fire trail \\
\hline C. formicifera 1 & NSW: Nattai National Park \\
\hline C. trapeziformis 1 & ACT: Black Mountain \\
\hline C. trapeziformis 2 & ACT: Black Mountain \\
\hline C. valida 1 & ACT: Lowden Road \\
\hline C. valida 2 & ACT: Black Mountain \\
\hline C. aff. plaricallate & NSW: Kanangra-Boyd National park \\
\hline
\end{tabular}

Table 1. Taxon and location of the Chiloglottis samples

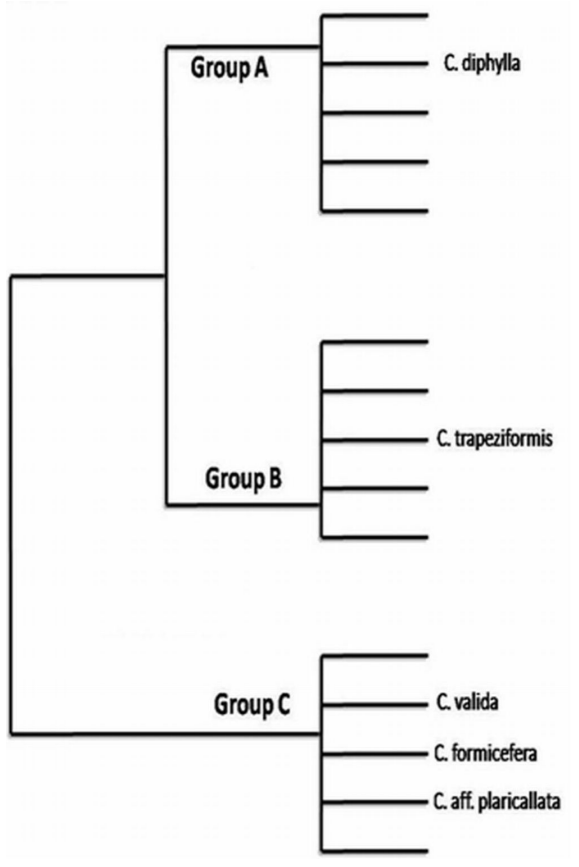

Figure 2. Simplified Chiloglottis phylogeny of the five sample species.

Source: Mant, Peakall \& Weston, 2005 
The PCR amplification was performed in CP-002 Palm Cycler (Corbett Research) with a touch-down thermal cycling program: $30 \mathrm{sec}$ denaturation at $94^{\circ} \mathrm{C}$ followed by 40 secs at $66^{\circ} \mathrm{C}$ for primer annealing and then 90 secs at $72^{\circ} \mathrm{C}$ for extension. The annealing temperature decreased by $4{ }^{\circ} \mathrm{C}$ at every cycle until it reached $54^{\circ} \mathrm{C}$, where it then reduced to $49^{\circ} \mathrm{C}$ for the last 10 cycles. Successful amplification of primer products in each sample was confirmed with gel electrophoresis $(2 \%, 20 \mathrm{~min} 130$ volts then $10 \mathrm{~min}$ at 145 volts $)$.

Purification of PCR products for sequencing followed ExoSAP-IT protocol (USB Corportation). The primers used for amplification were also used for sequencing, and all DNA fragments were sequenced in both directions. Sequencing reactions (20 $\mu \mathrm{l})$ contained $12.93 \mu \mathrm{l}$ milliQ H2O, $3.0 \mu \mathrm{l}$ X PCR buffer (Qiagen), $2 \mathrm{mM}$ of one primer, $0.32 \mu \mathrm{l} ; 0.75 \mu \mathrm{l}$ of ABI Big Dye (Perkin Elmer Boston, MA); and $50 \mathrm{ng} /$ $\mu \mathrm{l}$ of PCR Product, $3 \mu \mathrm{l}$. PCR sequence products were precipitated and purified with sodium acetate: ethanol followed by centrifuging and a $70 \%$ ethanol wash (x3).

\section{Sequence Analysis}

Alignment and editing of sequences was done in Geneious basic 4.6.4. PCR products for each sample were aligned to form a continuous sequence, except in $C$. formicifera which was constructed with only the reverse primer sequence for the second DNA segment as the forward primer sequence was corrupted. The PACPT sequences for each sample were aligned with the cDNA sequence from $C$. trapezformis to identify intron location, size and polymorphisms. Ambiguities in the identity of nucleotide bases were resolved with visual analysis of sequence chromatographs. All sequences were trimmed to 1645 base in length, commencing and ending at the same exon sequence. SNPs and insertions/deletions (indels) within the exons and introns of each sample were identified as species or clade specific. Extra nucleotide bases were classified as an insertion if present only in that species or clade. Likewise, if more than one clade shared the extra nucleotide bases, the species or clade without the extra nucleotide bases was classified as having a deletion. Phylogenetic trees were constructed using complete, trimmed sequences, as well as exon and intron only sequences (bootstrapping was not conducted). Finally, exon-only sequences of each sample were translated into amino acid sequences to identify the SNPs that may produce non-synonymous changes in the encoded amino acid sequence.

\section{Results}

Not only did the four primer pairs successfully amplify their intended products, producing a continuous $P A C P T$ gene sequence length (ranging from 1150 to 
1623 base pairs in length between species) in all of the Chiloglottis samples, but there was substantial interclade and interspecific genetic variation within the exon and intron sequence: rejecting the null hypothesis of no variation. Surprisingly, comparison of the $C$. trapeziformis cDNA sequence with nuclear sequence from the seven Chiloglottis samples (including 2 C. trapeziformis samples) revealed the location of 6 introns, 2 more than predicted (figure 3).

\section{A) Predicted introns}

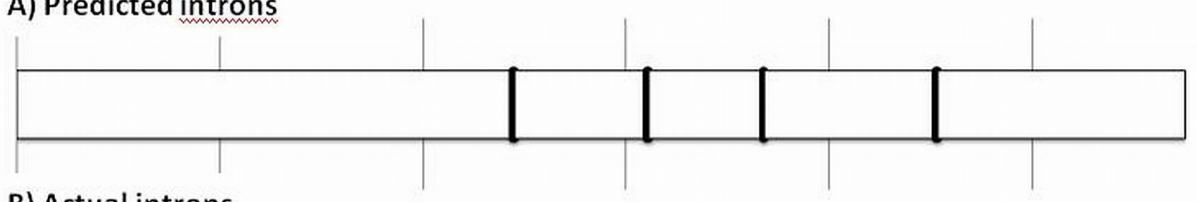

B) Actual introns

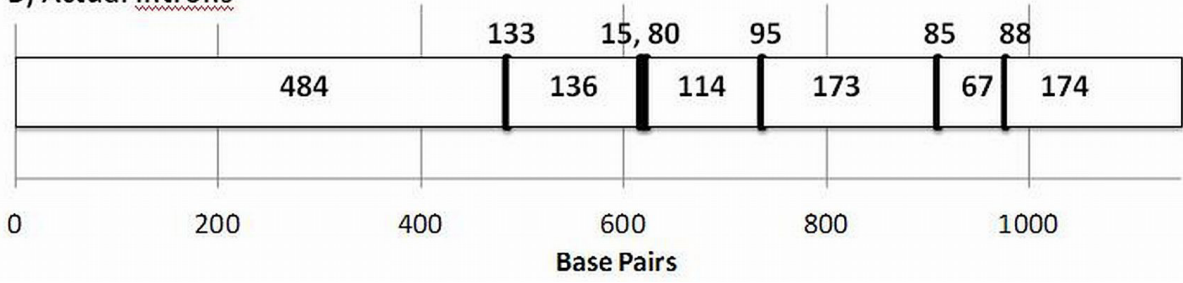

Figure 3. A) Four predicted introns in the PACPT sequence when compared to the complete Arabidopsis sequence. B) Actual position of 6 introns identified in the Chiloglottis consensus sequence.

The intron and exon-only phylogenetic trees showed identical genetic relatedness among samples, as did the phylogenetic tree constructed from the entire sequence (figure 4). The location and relatedness of species samples within the PACPT phylogenetic tree almost mimics that of Mant, Peakall, \& Weston's (2005) phylogeny constructed from comprehensive DNA fingerprinting methods (figure 2), except for $C$. formicera's position within the $C$. trapeziformis clade. This is due to the extremely high similarity (99.8\%) in the PACPT sequence between the two species. Such low genetic variation was not observed even between the two $C$. valida samples, who shared just $98.4 \%$ of their nucleotide bases. Similarity between the $C$. valida sampled from Black Mountain and $C$. aff. plaricallata was also very high, sharing $97.9 \%$ of their nucleotide bases. Overall, the PACPT gene sequence was fairly conserved between clades, with $91.3 \%$ of the sequenced nucleotide bases being shared amongst all seven Chiloglottis samples. 


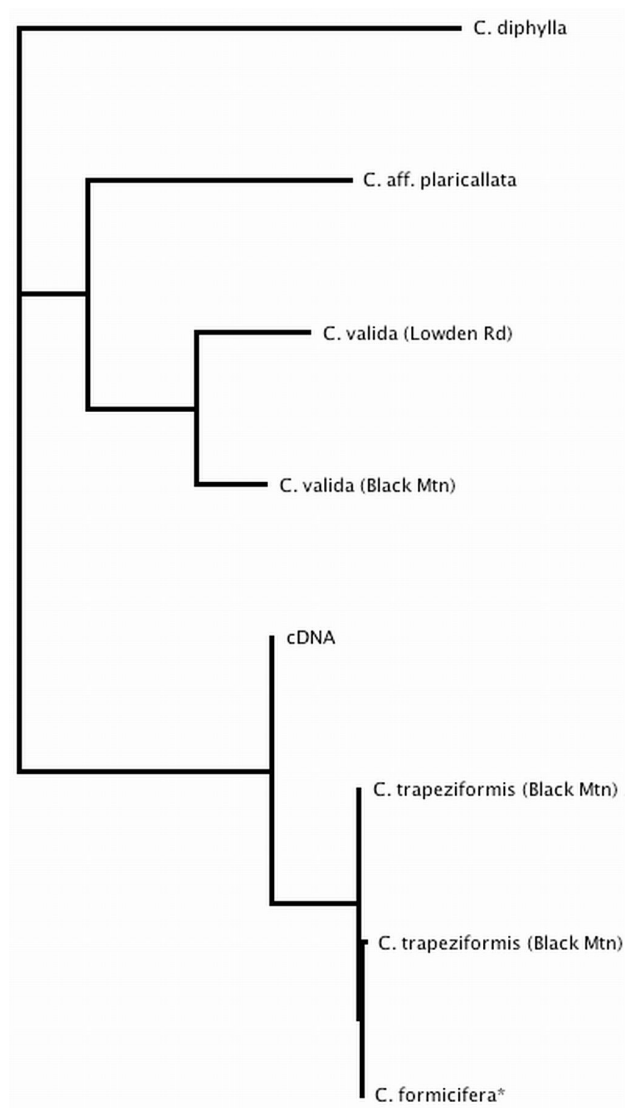

Figure 4. Phylogenetic tree constructed from ACPT gene sequences from seven Chiloglottis samples (cDNA is exon-only sequence from a C. trapeziformis)

Analysis of intron-only sequences revealed taxonomic differences in the distribution of variation, with $64 \%$ of variation accounting for differences between clades. Overall, $86 \%$ of nucleotide bases were shared across all seven samples with 49 SNPS and 5 indels distinguishing Chiloglottis species among the three clades (table $3 \&$ figure 5). There was no variation in the intron sequence between $C$. formicifera and $C$. trapeziformis samples (as previously mentioned), but there was surprising intra-species variation within the $C$. valida clade. The C. valida sampled from Black Mountain shared 7 SNPs and a 7bp deletion with the $C$. aff. plaricllate sample, which were not present in the $C$. valida sampled from Lowden Road. 


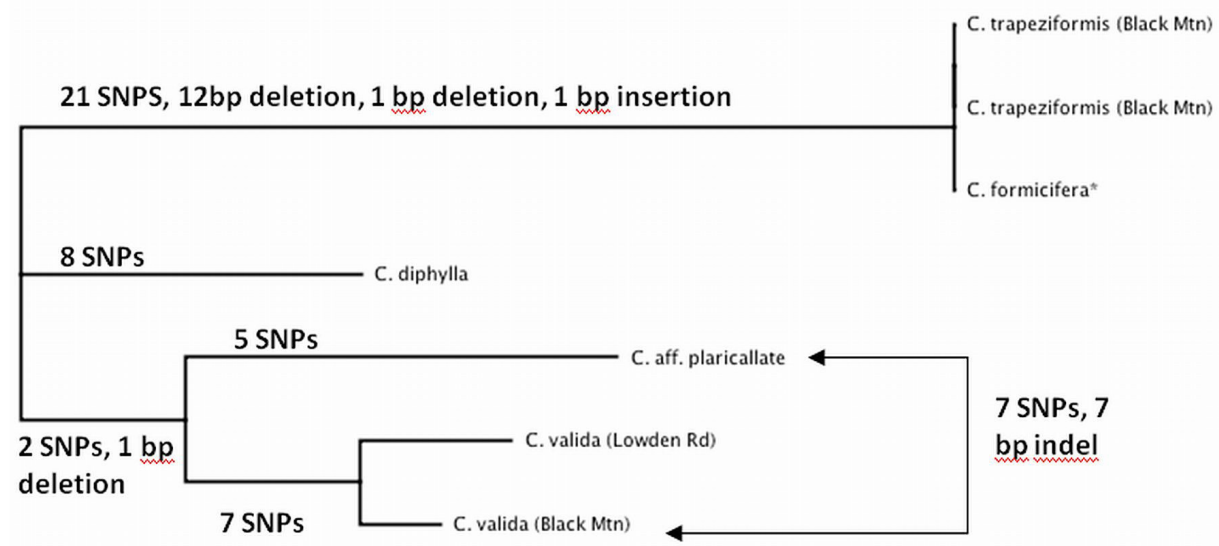

Figure 5. Intron-only phylogenetic tree illustrating the distribution of genetic variation within the intron sequences of the PACPT gene in seven Chiloglottis samples.

The distribution of genetic variation in the exon sequences was similar to that within introns, with $78 \%$ of the variation accounting for differences between clades, and each clade is being distinguishable by unique SNP combinations (although there were no indels within the exon regions) (figure 6). Again there was no species-defining genetic variation within the $C$. trapeziformis clade in the exon sequence, making it impossible to uniquely identify these two species based on these PACPT gene sequences alone. As expected, the exon sequences were less variable than the intron sequences, with $93.5 \%$ of nucleotides shared by all samples (this is due to the absence of indels within exon regions). Approximately $50 \%$ of all the SNPS within exons translate into nonsynonymous changes in the encoded amino acid (AA) sequence across the seven Chiloglottis samples, with each sample from the $C$. diphylla and $C$. valida clades encoding for at least 1 unique AA change (figure 6). 


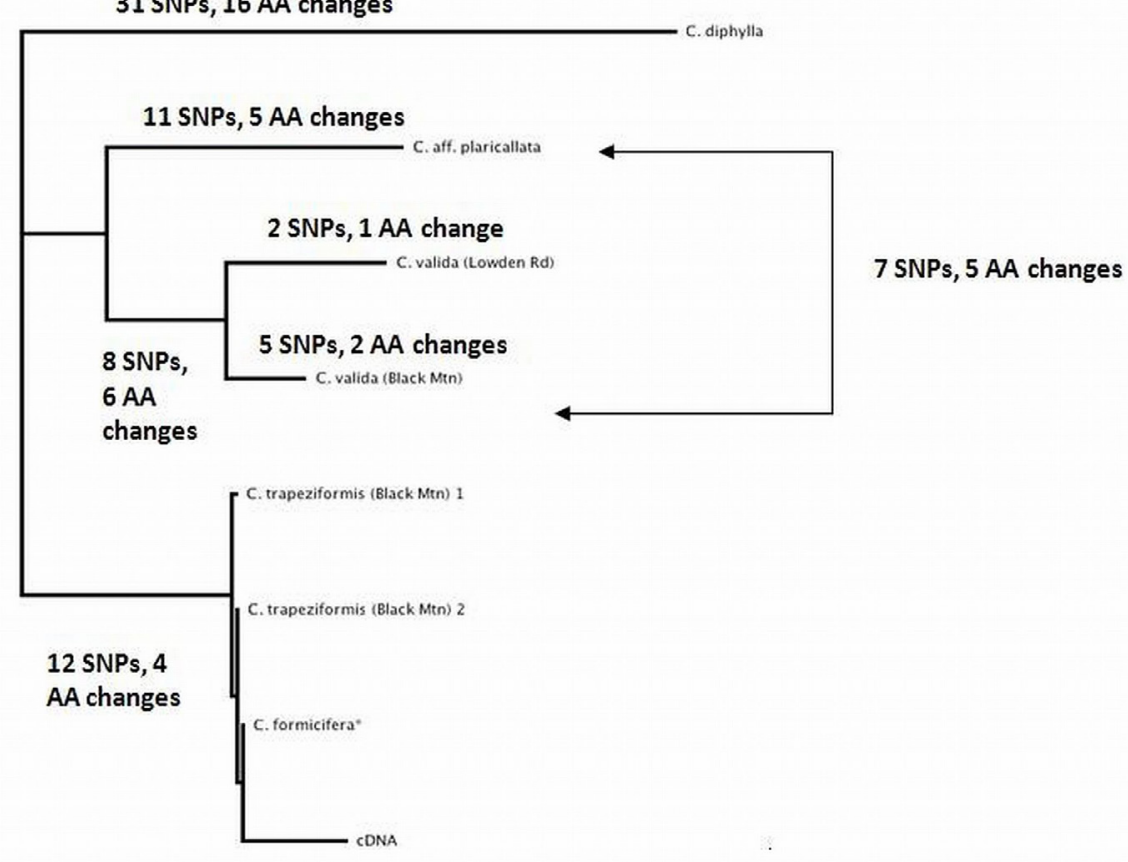

Figure 6. Exon-only phylogenetic tree illustrating the distribution genetic variation within the exon sequences of the PACPT gene in seven Chiloglottis samples.

\section{Discussion}

An analysis was conducted of the genetic variation in the exon and intron nucleotide sequences within the PACPT gene, which is found to be highly expressed in the pheromone-producing labellum of Chiloglottis orchids (Schiestl \& Peakall 2005). Genetic variation that codes for changes to the PACPT amino acid sequence were identified both among and within clades (figure 6). In addition, species-specific genetic variation within the (unexpressed) intron segments were mapped, thereby providing an additional genetic marker to complement existing phylogenetic markers and possibly aid in the identification of cryptic Chiloglottis taxa and other Orchideae (figure 5).

The high expression of the PACPT gene within the pheromone-producing labellum has lead researches to suspect that it may be involved in the production of pheromone compounds. As a metabolically essential protein that determines the length of plant fatty acids (Jones et al., 1995), it is certainly possible that $P A C P T$ plays a role in the production of the active compounds used to mimic pollinator sex pheromones across the Chiloglottis genera. As such, the high 
percentage $(50 \%)$ of SNPs identified within the expressed exon sequences that result in nonsynonymous changes in the encoded amino acid sequence of PACPT (at least 5 per species excluding $C$. formicifera) may create differences in the form and function of the protein between species and certainly between genetically divergent clades (figure 6). Unfortunately, the poorly understood effects of post-transcription and post-translation regulatory mechanisms (Campbell et al., 2006) on $P A C P T$ function prevent any conclusions as to how, and what degree, these identified SNPs might change the role of PACPT in the plants. However, given the variety of SNPs, and subsequent variation in the amino acid sequence within species (see $C$. valida figure 6 ), between species and particularly between clades, there is evidence to suggest that $P A C P T^{\prime}$ 's metabolic function may vary between Chiloglottis clades even if the protein is not involved in pheromone production.

Although exon variation was identified (figure 6), the level of conservatism within the coding regions of $P A C P T$ is high enough to allow four pairs of primers designed from $C$. trapeziformis cDNA sequence to successfully amplify the intended gene sequence $(1,645$ nucleotide bases) across the five Chiloglottis species. The conservation of the PACPT gene sequence is also illustrated in the way that its phylogeny mirrors the Chiloglottis phylogeny constructed from a more comprehensive AFLP fingerprinting method (Mant, Peakall \& Weston's (2005) ) (figure 2, 4 \& 5). Congruency between phylogeny and variation in sex pheromone compounds is reported in Lepidopteran moths (Lofsdedt \& Kozlov, 1996 as cited by Mant et al., 2002) and within some species of thyninne wasps (Mant et al., 2002). However there was one exception to this pattern; the sequence of $P A C P T$ in C. formicifera is identical to that of C. trapeziformis even though they are relatively distantly related species. While there is less certainty in the sequence of $C$. formicifera due to one forward primer segment being discarded, it is unlikely that the reverse sequence was misinterpreted. In addition, there was no cross-species contamination evident in other PCR products or the negative control. Lastly, increased species sampling could only reposition $C$. formicifera into its taxonomically correct $C$. valida clade if there is extremely high intra-species variation within the PACPT gene, which has not been obsevered within this study. Instead, it is likely that the PACPT gene is highly conserved between this two distantly related species, either due to pollinator-driven selection (Mant, Peakall, \& Schiestl, 2005) or other selection pressures on its metabolic functions (Jones et al., 1995).

The distribution of genetic variation was taxonomically unbalanced, with a high proportion of variation due to the differences between clades in both exons (78\%) and introns (64\%). Mant, Peakall \& Weston (2005) found similarly large genetic differences between the three Chiloglottis clades $(73 \%$ of the total variation) relative to species within the clades. The lack of genetic differentiation 
between species is suggested to be the result of consistent (or episodic) gene flow among closely related orchids (Mant, Peakall \& Weston, Schiestl 2005; Mant, Peakall \& Weston, 2005). Schiestl and Peakall (2005) demonstrated that different species of Chiloglottis (C. trapeziformis and $C$. valida) can indeed attract each others pollinators through joint production of the sex pheromone Chilogottone, thereby facilitating hybridization and gene flow between species. The joint attraction of even distantly related pollinators and subsequent gene flow between sympatric orchid species has also been supported in microsatellite studies in Ophrys orchids by Soliva \& Widmer (2003). Whether gene flow between species can account for the high genetic similarity in the PACPT gene among the sampled Chiloglottis species cannot be inferred from the present results. However, the genetic similarity of species within the spring and summer-flowering $C$. trapeziformis and $C$. valida clades, and their relatively higher genetic differentiation from the autumn-flowering $C$. diphylla clade, does match the predicted relatedness of cross-pollinating plants in accordance with their ecological circumstances (Mant, Peakall \& Weston, 2005)

Comparing variation in exons versus introns in the PACPT sequence at all taxonomic levels supports my prediction of higher genetic variation in intron segments; the Chiloglottis species samples shared less nucleotide bases and had five indels in their intron sequences compared to the exon segments (which had no indels difference between species) (figure 5 \& 6). However, further sampling and statistical tests would need to be done to confirm whether introns do have significantly higher variation than exons. Variation in the number of introns within the PACPT gene between Chiloglottis and Arabidopsis (figure 2) species is an important result, but not unusual as the two plants are not closely related (Campbell et al., 2006). Nevertheless, it does provide an additional measure of the genetic variation that exists within the $P A C P T$ gene in plants and may be useful for phylogenetic studies.

The identification of cryptic $C$. valida and other Chiloglottis subspecies to date has been based on the responses of pollinators to their unique chemical signatures (Bower \& Brown 2009), as have cryptic Ophrys species (Vela et al., 2007). However, the use of short intron sequences for identification of cryptic species would be valuable in many instances, particularly sympatric species that may attract multiple pollinators (Schiestl \& Peakall 2005). Aside from $C$. formicifera, I hypothesise that the four sampled species could be assigned to their correct taxonomic clade solely on species-specific variation within the intron sequence (varying from 2 to 21 SNPs and accompanying indels), based on multiple samples within the $C$. formicifera and $C$. valida clades and the overall genetic divergence of the $C$. diphylla clade (figure 5). Although cladespecific variation was observed in exon regions, the shorter length and higher proportion of variation within introns suggests that intron-based diagnostics 
would be ideal for identifying morphologically similar or cryptic Chiloglottis species (such as $C$. valida subsp., Bower \& Brown 2009). Future research would benefit from designing primers that target the diagnostic intron sequences in the sampled species followed by wide-spread testing in the Chiloglottis genera.

\section{Conclusion}

This study provides the first measure of the distribution of genetic variation in the PACPT gene sequence across the three main Chiloglottis clades. The high number of nonsynonymous changes to the encoded amino acid sequence of PACPT identified in four of the five Chiloglottis species sampled indicates that PACPT'S metabolic function may indeed vary within and between Chiloglottis clades. This genetic differentiation among species provides evidence in support of the hypothesis that $P A C P T$ is involved in the production of species-specific pheromone production. Future research will benefit by increasing the orchid sample size (including more taxa), as well conducting multiple extractions from each plant (to add consistency and statistical strength to the data). Continued differential analyses of $P A C P T$ expression between the labellum and other parts of the plant will also indicate whether $P A C P T$ is indeed expressed significantly higher in the pheromone-producing labellum than anywhere else in the plant; providing further evidence for the protein's potential role in the evolution and variation of pollinator sex pheromones in Chiloglottis orchids. An important secondary outcome from the design of novel primers for the partial sequencing of the PACP gene is the identification of species and clade-specific genetic variation. These genetic markers could be used for the identification of morphologically similar or cryptic species that so often characterise sexually deceptive genera (Mant, Peakall \& Weston, 2005; Bower \& Brown 2009). Aside from diagnostic purposes, the variability in these primer segments may complement existing markers in phylogenetic investigations in taxonomic groups with little genetic differentiation (Mant et al., 2002).

\section{Acknowledgments}

I would like to thank my project supervisors Rod Peakall, Christine Hayes and Michael Whitehead, lab associate Lee Clarke and course supervisor Celeste Linde, of the School of Botany and Zoology, Australian National University, for their support and assistance with both the laboratory and theoretical aspects of this project. 


\section{References}

Campbell NA, Reece JB, and Meyers N, (2006), Biology, 7th ed. Pearson Education: N.S.W. Australia.

Dafni A, Bernhardt P, (1990), Pollination of Terrestrial Orchids of Southern Australia and the Mediterranean Region - Systematic, Ecological and Evolutionary Implications, Evolutionary Biology, 24: 193-252.

Jones A, Maelor DH, Voelker TA, (1995), Palmitoyl-Acyl Carrier Protein (ACP) Thioesterase and the Evolutionary Origin of Plant Acyl-ACP Thioesterases, Plant Cell, 7: 359 -371.

Mant JG, Schiestl FP, Peakall R, and Weston PH, (2002), A Phylogenetic Study of Pollinator Conservatism Among Sexually Deceptive Orchids, Evolution, 56(5): $888-898$.

Mant J, Peakall R, and Schiestl FP, (2005), Does Selection on Floral Odor Promote Differentiation among Population and Species of the Sexually Deceptive Orchid Genus Ophrys?, Evolution, 59(7): 1449 -1463.

Mant J, Peakall R, and Weston PH, (2005), Specific pollinator attraction and the diversification of sexually deceptive Chiloglottis (Orchidaceae), Plant Systematics and Evolution, 253: 185-200.

Purdue Genomics Facility, (2010), cDNA sequence DiamondA2_cn0124, http://www.genomics.purdue.edu/ pmiguel/projects/Dudareva/ DiamondAntOrchid_1-8/DiamondAntOrchid.contigall.html [Accessed 20 November 2010].

Rozen S, Skaletsky H, (2000), Primer3 on the WWW for general users and for biologist programmers. In: Krawetz S, Misener S, (eds) Bioinformatics Methods and Protocols: Methods in Molecular Biology. Humana Press, Totowa, NJ: 365-386.

Schiestl FP, Peakall R, Mant J, Schulz C, Ibarra F, Frank F, (2003), The chemistry of sexual deception in an orchid-wasp pollination system, Science, 302: 437 -438 .

Schiestl FP, and Peakall R, (2005), Two orchids attract different pollinators with the same floral odour compound: ecological and evolutionary implications, Functional Ecology, 19: 674-680.

Soliva M, and Widmer A, (2003), Gene Flow Across Species Boundaries in Sympatric, Sexually Deceptive Ophrys (Orchidaceae) Species, Evolution, 57(10): $2252-2261$. 
Vela E, Tirard A, Renucci M, Suehs C M, and Provost E, (2007), Floral Chemical Signatures in the Genus Ophrys L. (Orchidacea): A preliminary Test of a New Tool for Taxonomy and Evolution, Plant Moleculuar Biology Reporter, 25(34): $83-97$. 